\title{
A methodology for direct exploitation of available information in the online model-based redesign of experiments
}

\author{
Riccardo De-Luca ${ }^{1, *}$, Federico Galvanin ${ }^{2}$, Fabrizio Bezzo ${ }^{1, *}$ \\ ${ }^{1}$ CAPE-Lab-Computer-Aided Process Engineering Laboratory \\ Department of Industrial Engineering, Università di Padova \\ via Marzolo 9 I-35131 Padova PD (Italy) \\ ${ }^{2}$ Department of Chemical Engineering, University College London \\ Torrington Place, London WC1E 7JE, United Kingdom
}

Accepted to: Computers and Chemical Engineering

Copyright @ 2014 R. De-Luca, F. Galvanin, F. Bezzo

UNPUBLISHED

No parts of this paper may be reproduced or elsewhere used without the prior written permission of the authors

Original manuscript submitted on: 29 July 2015

Revised manuscript submitted on: 16 March 2016

\begin{abstract}
Online model-based design of experiments techniques were proposed to exploit the progressive increase of the information resulting from the running experiment, but they currently exhibit some limitations: the redesign time points are chosen "a-priori" and the first design may be heavily affected by the initial parametric mismatch.

In order to face such issues an Information Driven Redesign Optimization (IDRO) strategy is here proposed: a robust approach is adopted and a new design criterion based on the maximisation of a target profile of dynamic information is introduced. The methodology allows determining when to redesign the experiment in an automatic way, thus guaranteeing that an acceptable increase in the information content has been achieved before proceeding with the intermediate estimation of the parameters and the subsequent redesign of the experiment. The effectiveness of the new experiment design technique is demonstrated through two simulated case studies.
\end{abstract}

Keywords: Online model-based design of experiments, robust design, model identification, parameter estimation

\footnotetext{
*Corresponding authors: E-mail addresses: fabrizio.bezzo@unipd.it
} 


\section{Introduction}

Model-based design of experiments (MBDoE) techniques (Pukelsheim, 1993) represent a valuable tool for the rapid assessment and development of mathematical models at different levels of the model building procedure (Asprey and Macchietto, 2000), allowing for the maximization of the experimental information in order to reduce time and costs of the model identification task. The effectiveness of the conventional iterative MBDoE procedure (Franceschini and Macchietto, 2008) has been proved in a large variety of applications (Prasad and Vlachos 2008; Galvanin et al. 2009a; Chakrabarty et al. 2013, Vanderlinden et al. 2013) but it is greatly limited by the fact that the design activity is affected by the initial available estimates of parameter values; therefore high uncertainty on these values can severely affect the efficiency of the experimental design (Körkel et al., 2004). In fact, several approaches were proposed in order to overcome this problem. For example, Houska et al. (2015) proposed an iterative optimal design method that consists of a modified A-criterion weighting the terms information matrix trace calculated through an ad-hoc algorithm. As stated by Mehra (1974), an efficient design procedure may be based on the exploitation of the information as soon as it is generated by the running experiment through an online adaptive input design AID) strategy. AID strategies have been proposed and applied to linear stochastic control system (Lindqvist and Hjalmarsson, 2001; Gerencser et al., 2009), where the design task is solved over a preset time horizon after which a new measurement is taken and, consequently, a new estimation is acquired. Extension of this procedure to nonlinear dynamic system is quite a recent achievement (Stigter et al., 2006) and an online model-based redesign of experiments (OMBRE) approach has been recently proposed as a natural expansion of AID strategies (Galvanin et al., 2008, 2009). In OMBRE the manipulated variables dynamic profiles and the sampling points allocation are updated by performing one or more intermediate experiment designs (redesigns). The efficiency of this technique has been tested for a wide range of cases by different research groups both for model validation (Barz et al., 2013) and model discrimination 
issues (Schenkendorf and Mangold, 2013), providing a very efficient usage of measurement data and a great improvement with respect to conventional MBDoE techniques. The OMBRE approach has been also recently extended to systems where disturbances and systematic errors may be present by using model updating policies including disturbance estimation procedures which are embedded within the OMBRE strategy (Galvanin et al., 2012).

Unfortunately, OMBRE technique is affected by some limitations, too: firstly, the redesign policy is decided a priori by the user, without any rational criterion related to the achievable information; secondly, OMBRE approach is still affected by the initial parameter uncertainty, especially until a first redesign is done.

In this work a novel information-driven redesign optimisation (IDRO) is presented, where a robust design approach is applied to the online redesign procedure with the purpose of determining when to redesign the experiment in an automatic and robust way. IDRO is based on a new design concept, based on the maximisation of a target profile of the dynamic information profile, which guarantees a reliable increase in the information content before proceeding with the intermediate estimation of the parameters and the subsequent redesign of the experiment. Furthermore, the technique is much less affected by the negative effects of parametric uncertainty, with great benefit in terms of robustness of the whole redesign procedure. The applicability to nonlinear dynamic systems is demonstrated through two simulated case studies: the first one is related to the identification of a fermentation bioreactor model, the second one is based on the identification of a physiological model which describes glucose homeostasis.

\section{Optimal design of experiments: methodology}

In this paragraph the mathematical and statistical background required to understand the basic features of the conventional MBDoE procedure for parameter estimation is firstly described; then 
OMBRE approach is defined in mathematical terms; finally the new proposed method IDRO is presented in detail before comparing the three methods through the two simulated case studies.

\subsection{Conventional MBDoE}

A conventional MBDoE procedure (Asprey and Macchietto, 2000; Körkel et al., 2004) aims at decreasing the model parameter uncertainty region by acting on the $n_{\varphi}$-dimensional experiment design vector $\boldsymbol{\varphi}$ and solving the following nonlinear optimisation problem:

$\boldsymbol{\varphi}^{\mathbf{o p t}}=\arg \min _{\boldsymbol{\varphi}}\left\{\psi\left[\mathbf{V}_{\theta}(\boldsymbol{\theta}, \boldsymbol{\varphi})\right]\right\}=\arg \min _{\boldsymbol{\varphi}}\left\{\psi\left[\mathbf{H}_{\theta}^{-1}(\boldsymbol{\theta}, \boldsymbol{\varphi})\right]\right\}$

subject to

$$
\begin{aligned}
& f(\dot{\mathbf{x}}(t), \mathbf{x}(t), \mathbf{u}(t), \mathbf{w}, \boldsymbol{\theta}, t)=0, \quad \hat{y}=h(\mathbf{x}) \\
& \mathbf{C}=\mathbf{x}(t)-\mathbf{G}(t) \leq 0 \\
& \boldsymbol{\varphi}_{i}^{l} \leq \boldsymbol{\varphi}_{i} \leq \boldsymbol{\varphi}_{i}^{u}, \quad i=1 \ldots n_{\varphi}
\end{aligned}
$$

with the set of initial conditions $\mathbf{x}(0)=\mathbf{x}_{0}$. In (1) $\mathbf{V}_{\theta}$ is the variance-covariance matrix of model parameters and $\mathbf{H}_{\theta}$ corresponds to its approximated inverse, the dynamic Fisher information matrix; $\mathbf{x}(t) \in \mathfrak{R}^{N_{x}}$ is the time-dependent state variables vector, $\mathbf{u}(t) \in \mathfrak{R}^{N_{u}}$ and $\mathbf{w} \in \mathfrak{R}^{N_{w}}$ are, respectively, the time-dependent and time-invariant control variables (manipulated inputs), $\boldsymbol{\theta} \in \mathfrak{R}^{N_{\theta}}$ is the model parameters set, and $t$ is time. The symbol ${ }^{\wedge}$ is used to specify the estimated value of a variable (or a set of variables): for example, $\mathbf{y}$ represents the vector of measured values of the outputs, while $\hat{\mathbf{y}}$ is the vector of the corresponding values estimated by the model. In equation (4) $\mathrm{C}$ is the $N_{c^{-}}$ dimensional set of constraint functions expressed through the set $\mathbf{G}(t) \in \mathfrak{R}^{N_{C}}$ of active constraints on state variables. Equation (5) represents the $n_{\varphi}$-dimensional set of constraints on design variables, usually expressed by lower (superscript $l$ ) and upper (superscript $u$ ) bounds on each components of the experiment design vector $\boldsymbol{\varphi}$, constraining the design to a hyperrectangular subspace of the overall design space $\mathfrak{R}^{n_{\varphi}}$. The experiment design vector $\boldsymbol{\varphi}$ is defined as: 
$\boldsymbol{\varphi}=\left[\mathbf{y}_{0}, \mathbf{u}(t), \mathbf{w}, \mathbf{t}^{s p}, \tau\right]^{T}$

It consists of the $N_{y}$-dimensional set of initial conditions for the measured variables $\mathbf{y}_{0}$, the manipulated input variables $\mathbf{u}(t)$ and $\mathbf{w}$, the total duration of the experiment $\tau$ and the $N_{s p^{-}}$ dimensional set of output variables sampling times $\mathbf{t}^{s p}$.

Function $\psi$ in (1) is an assigned metric of the variance-covariance matrix of model parameters $\mathbf{V}_{\theta}$ and represents the design criterion adopted to maximise the expected information content of the experiment as predicted by the model; the most common design criteria are the alphabetical ones, i.e. A-, D-, E-optimal criteria which focus on trace, determinant and maximum eigenvalue of $\mathbf{V}_{\theta}$ minimization, respectively (Pukelsheim, 1993). The dynamic information matrix $\mathbf{H}_{\theta}$ for a single experiment is usually expressed by a discrete dynamic form of the Fisher information matrix (Bard, 1974). According the notation proposed by Zullo (1991), $\mathbf{H}_{\theta}$ is here defined as:

$$
\mathbf{H}_{\theta}(\boldsymbol{\theta}, \boldsymbol{\varphi})=\mathbf{H}_{\theta}^{\mathbf{0}}+\sum_{k=1}^{N_{s p}} \sum_{i=1}^{N_{y}} \sum_{j=1}^{N_{y}} s_{i j}\left[\frac{\partial \hat{y}_{i}\left(t_{k}\right)}{\partial \theta_{l}} \frac{\partial \hat{y}_{j}\left(t_{k}\right)}{\partial \theta_{m}}\right]_{l, m=1 \ldots N_{\theta}}=\mathbf{H}_{\theta}^{\mathbf{0}}+\sum_{k=1}^{N_{s p}} \mathbf{M}_{k}
$$

In (7) $s_{i j}$ is the $i j$-th element of the inverse of the $N_{y} \times N_{y}$ measurement error covariance matrix $\boldsymbol{\Sigma}_{\mathbf{y}}$, $\mathbf{M}_{k}$ represents the amount of information that can be obtained at the $k$-th sample and $\mathbf{H}_{\theta}^{0}$ is the preliminary information matrix, based on the preliminary statistics about the parametric system at the beginning of the experimental campaign.

The conventional MBDoE (Franceschini and Macchietto, 2008) can be described as an iterative loop procedure generally based on the following steps:

1. get prior knowledge on the parametric set value (and its related uncertainty $\boldsymbol{\Sigma}_{\theta}$ );

2. choose an optimal design criterion and design the experiment;

3. start the experiment;

4. at the end of the experimental run, estimate the model parameters. 
5. if the desired estimation quality is not reached by the end of the experiment, design a new experiment, based on the model parameters estimated in step 4.

\subsection{Online model-based redesign of experiments (OMBRE)}

When OMBRE procedure is used, intermediate parameter estimations are carried out at specific updating times while the experiment is running in order to exploit the information obtained and use it to partially design the remaining part of the test. The experimental run is thus split into subexperiments, within which the experiment decision variables are distributed according to the "a priori” chosen redesign strategy (Galvanin et al., 2009). Following this approach, the global design vector of the experiment $\varphi$ can therefore be rewritten as:

$\boldsymbol{\varphi}=\left[\boldsymbol{\varphi}_{1}, \boldsymbol{\varphi}_{2}, \ldots, \boldsymbol{\varphi}_{j}, \ldots, \boldsymbol{\varphi}_{n^{u p}+1}\right]^{\mathrm{T}}$

where $n^{u p}$ is the number of updating times, and $\boldsymbol{\varphi}_{j}$ is the design vector before the $j$-th update; each component $\boldsymbol{\varphi}_{j}$ of $\boldsymbol{\varphi}$ could have a different dimension in terms of number of discretised control variables and/or sampling points. Obviously, $\boldsymbol{\varphi}_{1}$ will be the only component including the initial values of measured variables.

For each redesign activity an optimisation problem acting on the $j$-th component of $\varphi$ is conducted in the corresponding time frame; in particular, the optimality condition for $\boldsymbol{\varphi}_{j}$ is given by

$$
\boldsymbol{\varphi}_{j}^{\text {opt }}=\arg \min _{\boldsymbol{\varphi}_{j}}\left\{\psi\left[\mathbf{V}_{\theta \mid j}\left(\boldsymbol{\theta}, \boldsymbol{\varphi}_{j}\right)\right]\right\}=\arg \min _{\boldsymbol{\varphi}_{j}}\left\{\psi\left[\mathbf{H}_{\theta \mid j}^{-1}\left(\boldsymbol{\theta}, \boldsymbol{\varphi}_{j}\right)\right]\right\},
$$

where the information acquired by the $j$-th redesign can be expressed as a partial contribute to the total dynamic information matrix:

$$
\mathbf{H}_{\theta \mid j}\left(\boldsymbol{\theta}, \boldsymbol{\varphi}_{j}\right)=\tilde{\mathbf{H}}_{\theta}\left(\boldsymbol{\theta}, \boldsymbol{\varphi}_{j}\right)+\sum_{k=0}^{j-1} \tilde{\mathbf{H}}_{\theta \mid k}\left(\boldsymbol{\theta}, \boldsymbol{\varphi}_{k}\right)+\boldsymbol{\Sigma}_{\theta}^{-1}=\tilde{\mathbf{H}}_{\theta}\left(\boldsymbol{\theta}, \boldsymbol{\varphi}_{j}\right)+\mathbf{L}
$$

The constant term $\mathbf{L}$ consists on the sum between the preliminary information matrix $\boldsymbol{\Sigma}_{\theta}^{-1}$ and the information acquired before the $j$-th redesign. The symbol $(\sim)$ indicates that the information matrix 
refers to a single updating interval, and $\widetilde{\mathbf{H}}_{\theta \mid 0}$ is the null matrix. At each given updating time the information is obtained by executing online a parameter estimation session followed by a redesign of the remaining part of the experiment.

The detailed OMBRE procedure is therefore based on the following steps (Figure 1):

1. get prior knowledge on the parametric set value (and its related prior variance-covariance $\boldsymbol{\Sigma}_{\theta}$ );

2. choose an updating strategy and design the first sub-experiment by calculating (9);

3. start the experiment;

4. if an updating time is reached, estimate the model parameters:

a. if a statistically sound parameter estimation is achieved, then stop the redesign procedure (and possibly the experiment itself); otherwise

b. redesign the remaining part of the experiment by using (9) with the update on dynamic information given by (10); implement the design in the running experiment, and go to step 4.

5. if a satisfactory parameter estimation is not achieved by the end of the experiment, design a new experiment.

Note that a possible parametric mismatch can be managed by OMBRE only by adjusting the model parameters according to the available observations. The optimality condition and the feasibility conditions will be evaluated within each time frame according to the current value of model parameters.

\subsection{Information-driven redesign optimisation (IDRO) for model-based design of}

\section{experiments}

In OMBRE methodology the updating strategy is chosen a priori by the user without taking into account any rational criterion related to the achievable information; in order to overcome this issue a new updating strategy is here proposed including: i) the optimisation of the information obtainable 
from the experiment based on a target information; ii) the identification of the best updating time to redesign.

The design task, especially at the beginning of the procedure, may be affected by an initial parametric mismatch. Consequently, in order to preserve the quality of the experiment by poor starting values of the parameters, a robust optimal design approach (Asprey and Macchietto, 2002) based on the expected value of a measurement function of the predicted information is implemented.

IDRO methodology is essentially based on a robust A-optimal criterion applied for the worst model parameter, by exploiting one of the most significant statistical indices used to evaluate the parameter estimation precision: the $t$-value. This index is strictly linked to the information that can be gained during the experimental run, as can be observed from the following equation:

$t_{i}=\frac{\theta_{i}}{t\left(\frac{1}{2}+\frac{1-\alpha}{2}, N_{s p}-N_{\theta}\right) \cdot \sqrt{v_{i i}}}, \quad i=1, \ldots, N_{\theta}$,

where $t_{i}$ is the $t$-value related to the $i$-th model parameter, $v_{i i}$ is the $i i$-th term of the parametric variance-covariance matrix, $t(\cdot)$ is the $t$-value distribution with a $[1 / 2+(1-\alpha) / 2] \%$ confidence level and $\left(N_{s p}-N_{\theta}\right)$ degrees of freedom, where $\alpha$ is the statistical level of significance. As can be inferred from the previous equation, each $t_{i}$ value increases at each sampling time both for the information acquired by the sample itself in terms $v_{i i}$ of and for the variation of the $t$-distribution due to the stepwise increase of $N_{s p}$ at each sampling point.

A statistically sound parameter estimation is reached for all the model parameters if each parametric $t$-value is greater than the $(1-\alpha) \%$ confidence reference $t$-value $\left(t_{1-\alpha}^{r e f}\right)$, which is defined as:

$t_{1-\alpha}^{r e f}=t\left(1-\alpha, N_{s p}-N_{\theta}\right)$.

The IDRO method consists therefore in conducting the experiment in order to overcome the $t_{1-\alpha}^{\text {ref }}$ value threshold by the end of experiment, through the online redesign of the experiment. The 
objective function has been defined as the time integral of the difference between the updating time for each redesign and the total duration of the experiment ${ }^{\dagger}$. Moreover, the optimisation is constrained by imposing that, for each redesign task, the predicted precision of all parameters should be greater than a preset threshold.

The mathematical expression of the optimal values of each $\varphi_{j}$ component of the $\varphi$ design vector, as defined in equations (6), is therefore:

$$
\boldsymbol{\varphi}_{j}^{\mathrm{opt}}=\arg \max _{\varphi_{j}}\left[\int_{t^{u p, j}}^{\tau}\left(t-t^{u p, j}\right) d t\right]
$$

$C=\underset{\theta \in \Theta}{E}\left\{\max _{i=1: N_{\theta}}\left[\left.w_{j-1} \cdot t_{1-\alpha}^{r e f}\right|_{t=t^{u p, j}}-\left.t_{i}\right|_{t=t^{u p, j}}\right]\right\}=0$,

where $t$ is the time variable, $t^{u p, j}$ is the $j$-th updating time, $w_{j-1}$ is a weight corrector that corresponds to the minimum fraction of the $t_{1-\alpha}^{r e f}$ to be reached by each parametric $t$-value by the end of the $j$-th updating time and $E(\cdot)$ is the expected value of the function in brackets, evaluated in the parameter domain $\Theta$. In practice, for each sub-experiment, $\varphi_{j}^{\text {opt }}$ corresponds to the design vector that allows minimizing both a specific percentage of deviation between the $t_{1-\alpha}^{r e f}$ and the minimum $t_{i}$ and the value of the time $t^{u p, j}$ necessary to achieve this result.

The robustness of IDRO is based on the fact that each $\varphi_{j}^{\text {opt }}$ is calculated by evaluating the constraint defined in (14), which is an average value obtained through a stochastic simulation with $N_{s c}$ scenarios. Each scenario represents the model response at different $\theta$ values, which are randomly sampled in the parameter domain $\boldsymbol{\Theta}$ of the parameter covariance matrix by assuming a normal distribution $N\left(\hat{\theta}_{i}, \hat{\sigma}_{\theta i}\right)$, where $\hat{\theta}_{i}$ is the estimate of the $i$-th parameter and $\hat{\sigma}_{\theta i}$ is the standard

\footnotetext{
${ }^{\dagger}$ The integral formulation has been chosen in order to amplify the effect of the time distance between $t^{\text {up. } j}$ and $\tau$. Alternative formulations are possible.
} 
deviation of $\hat{\theta}_{i}$. In order to follow the logic of an A-optimal MBDoE, only the diagonal elements $\hat{\sigma}_{\theta i}^{2}$ of the variance-covariance matrix of model parameters are considered during the sampling procedure (i.e. covariance elements are ignored). Although this is an approximation, it has been verified that this choice does not affect the quality of the sampling in a significant way. The parametric set for each scenario has been randomly generated by considering only the diagonal terms of the parametric variance-covariance matrix ("diagonal" approach). This simplification is justified by the fact that the average and the standard deviation values of the sample distribution obtained through the "diagonal" approach only differ by $1 \%$ and $10 \%$, with respect to the case where all the terms of the variance-covariance matrix are included. Furthermore, it should be noted that $\hat{\theta}_{i}$ and $\hat{\sigma}_{\theta i}$ values are updated at each $t^{\text {up. } j}$ in order to take into account the results obtained through the parameter estimations conducted at the end of each sub-experiment, and the sampling effect is noticeable only at the very beginning of the design procedure (i.e. in the first design).

The logic of IDRO iterative procedure is illustrated in Figure 2. The picture simulates the hypothetical behaviour of IDRO approach for a two-parameters model. The red solid stepwise profile represents the weighted reference $t$-value profile, whose value decreases after each sampling point has been collected (initially the profile is constant to a preset value since no distribution can be computed for $\left.\left(N_{s p}-N_{\theta}\right)<0\right)$. The black solid and dashed stepwise lines represent the time profiles of the $t$-values for the two model parameters. These profiles will increase after each sampling point is collected as soon as additional information on parametric variability is acquired. Let us assume that in this case the dashed line is related to the most difficult parameter to be estimated (i.e. the parameter with minimum $t$-value); after fixing a weight $w_{0}$ for the first subexperiment, the IDRO optimisation (Eq. 13-14) will minimise the time required for the minimum $t$ value to become larger than the target $w_{0} \cdot t_{1-\alpha}^{\text {ref }}$ value; the optimal updating time $\left(t^{\text {up, },}\right)$ will be evaluated accordingly. Note that weight $w_{0}$ is initially introduced to set a minimum level of 
information to be reached within the first update; as will be shown in the actual algorithm, this weight is automatically adjusted to guarantee that the first update does not exploit all the available experimental capability in terms of variations on the input variables (i.e. the maximum number of switches on $\mathbf{u}(t)$, which is set a priori and typically depends on the equipment characteristics) as well as in terms of samples (i.e. the maximum number of measurements that can be taken, which is also set a-priori and depends on the experimental settings and on the cost and complexity of measurements). In other words, the procedure is built is such a way that at least one update is always carried out and that the remaining part of the experiment can still be designed according to an optimum criterion.

The experiment then starts and will be run till $t=t^{\text {up, }, 1}$, time at which a parameter estimation is carried out and a new (larger) weight $w_{1}$ for $t_{1-\alpha}^{\text {ref }}$ is chosen. From this point on, an iterative procedure is implemented until the end of the experiment is reached (in terms of time $\tau$ ) or $t_{1-\alpha}^{\text {ref }}$ is achieved (i.e. $w_{j} \geq 1$ ) or there is no experimental design capacity in terms of measurements or input switches.

The detailed IDRO procedure (Figure 3) is therefore based on the following steps:

1. get prior knowledge on the parametric set value (and its related uncertainty $\boldsymbol{\Sigma}_{\theta}$ ) and set $j=1$, where $j$ is the counter index of the sub-experiment to be designed;

2. set $w_{0}$, i.e. fix the minimum amount of information, that has to be reached by all the $t$-values $\left(t_{i}\right)$ at the end of the first sub-experiment, defined by $w_{0} \cdot t_{1-\alpha}^{r e f}$;

3. design the first sub-experiment from equations $(13,14)$; 
4. check if $t^{\text {up } j}<\beta \cdot \tau, N_{s w, j}<\gamma \cdot N_{s w}$ or $N_{s p, j}<N_{s p, j-1}+\delta \cdot N_{s p}$ are satisfied ${ }^{\dagger} ; \beta, \gamma$ and $\delta$ values are a priori set by the user in order to guarantee both sufficient time and a sensible number of switching levels and sampling points for the following sub-experiment/s;

a. if all conditions are satisfied, carry out the sub-experiment, estimate the model parameters and go to step 5 .

b. otherwise, set $w_{0}=k_{\varepsilon} w_{0}$ and go to step 3. $k_{\varepsilon}$ represents a preset reduction coefficient that allows to decrease the initial value assumed for $w_{0}$ during the iterative procedure;

At the end of the first sub-experiment, the following iterative procedure is used:

5. $\operatorname{set} j=j+1$;

6. set $w_{j}=k_{w} w_{j-1}$, where $k_{w}$ is the incremental coefficient. This index, whose value is preset by the user, represents the relative difference between the optimal weight $w_{j-1}$ used for the design of the $j-1$ sub-experiment and the initial tentative weight $w_{j}$ tested at the beginning of the optimisation loop for the $j$ sup-experiment;

7. design the $j$-th sub-experiment as described in $(13,14)$;

8. check if $t^{\mathrm{up}, j} \leq \tau$;

a. if true, go to step 9;

b. otherwise, set $w_{j}=k_{\varepsilon} w_{j}$ and go to step 7;

9. run the $j$-th sub-experiment (till $t^{\mathrm{up} \cdot j}$ ) and estimate the model parameters

10. if $w_{j-1}<1$ and/or $t^{\text {up }, j}<t^{\text {up. }, j-1}+\beta \cdot\left(\tau-t^{\text {up }, j-1}\right)$ and/or $N_{s w, j}<\gamma \cdot\left(N_{s w}-N_{s w, j-1}\right)$ and/or $N_{s p, j}<\delta \cdot\left(N_{s p}-\right.$ $\left.N_{s p, j-1}\right)$

a. then go to step 5;

\footnotetext{
* As anticipated above, this condition is required to ensure that one update is carried out and still the remaining part of the experiment can be designed in an optimal way. In other words, we do not want that: a) the update is scheduled too close to the end of the experiment; b) there is no further possibility to excite the system (no enough switches on input variables); c) there is no possibility to collect a reasonable additional number of samples. Note that parameters $\beta, \gamma$ and $\delta$ are the only ones that are indeed set by the users and somewhat reflects his/her knowledge on the experimental facility and the viability of performing portions of experiment.
} 
b. otherwise, stop;

11. if a satisfactory parameter estimation is not achieved, design a new experiment.

The target $\mathrm{t}$-value profile definition depends on the values assumed by $k_{\varepsilon}$ and $k_{w}$. As practical guidelines, we suggest:

- $k_{\varepsilon}$ should be lower than 1 , in order to have a progressive decrease of $w_{0}$ at each step of the loop: this allows testing the design feasibility without sacrificing the maximum achievable information at each design/redesign task;

- $k_{w}$ should be higher than $1 / w_{0}$. In this way $w_{j}$ can be higher than 1 soon after the second redesign of the experiment.

Two simulated case studies are examined in order to compare, in terms of parametric estimation precision at the end of the experimental run, standard MBDoE and OMBRE approaches with the newly proposed IDRO methodology; the two case studies differ in terms of number of measured responses, simulated experimental error on measured variables, and for the absence/presence of active constraints on the state variables. For standard MBDoE and OMBRE designs A-criterion is used because of its similarity with IDRO approach, which is based on the diagonal terms of the variance-covariance matrix. This will allow a more fair comparison between different criteria. The $\mathrm{gPROMS}^{\circledR}$ software has been adopted for designing, modelling and simulation purposes, as well as to conduct the "in silico" experiments and to analyse the results coming from them through its Parameter Estimation entity. The NLPSQP solver has been used for the optimisation/design task; it employs a sequential quadratic approach (SQP) method for the solution of a nonlinear programming (NLP) problem. gPROMS $^{\circledR}$ software is used in conjunction with external subroutines written in Fortran via Foreign Object Interface in order to implement the inversion of the information matrix and calculate the dynamic $t$-value profiles required by the IDRO approach. 


\section{Case study 1: fermentation bioreactor}

Conventional MBDoE, OMBRE and IDRO approaches are here compared and applied to a model that simulates a biomass fermentation process (Espie and Macchietto, 1989; Munack and Posten, 1989). The model consists of the following set of equations:

$$
\begin{aligned}
& \frac{d x_{1}}{d t}=\left(r-u_{1}-\theta_{4}\right) \cdot x_{1} \\
& \frac{d x_{2}}{d t}=-\frac{r x_{1}}{\theta_{3}}+u_{1}\left(u_{2}-x_{2}\right) \\
& r=\frac{\theta_{1} x_{2}}{\theta_{2}+x_{2}} \\
& \hat{y}_{i}=x_{i} \quad i=1,2
\end{aligned}
$$

where $x_{1}$ is the biomass concentration $(\mathrm{g} / \mathrm{L}), x_{2}$ is the substrate concentration $(\mathrm{g} / \mathrm{L}), u_{1}$ is the dilution factor $\left(\mathrm{h}^{-1}\right)$, and $u_{2}$ is the substrate concentration in the feed $(\mathrm{g} / \mathrm{L})$. The achievable conditions that characterise the experimental tests are the dilution factor $u_{1}$ (range $0.05 \div 0.20 \mathrm{~h}^{-1}$ ) and the substrate concentration in the feed $u_{2}$ (range $5 \div 35 \mathrm{~g} / \mathrm{L}$ ). These manipulated inputs are approximated by piecewise constant profiles over $N_{s w}=6$ switching intervals. The initial biomass concentration $x_{1}^{0}$ is allowed to assume values in the range $1 \div 10 \mathrm{~g} / \mathrm{L}$, whereas the substrate concentration $x_{2}^{0}$ is set to 0 $\mathrm{g} / \mathrm{L}$, respectively. It is assumed that both $x_{1}$ and $x_{2}$ can be measured during the experiment (i.e. $\mathbf{y}=$ $\left[\begin{array}{ll}x_{1} & x_{2}\end{array}\right]^{\mathrm{T}}$ ) and that its total duration is $\tau=48 \mathrm{~h}$. Measurements are affected by Gaussian noise with zero mean and variance-covariance matrix given by:

$$
\boldsymbol{\Sigma}_{y}=\left[\begin{array}{cc}
0.01 & 0 \\
0 & 0.05
\end{array}\right] \text {. }
$$

The "real" system is assumed to be characterised through the parameter set $\boldsymbol{\theta}=\left[\begin{array}{ll}0.310 & 0.180 \\ 0.550\end{array}\right.$ $0.050]^{\mathrm{T}}$. It is then assumed that the initial parameter estimates are affected by a $\pm 90 \%$ relative error with respect to the true parameter values (in this case, $\boldsymbol{\theta}^{0}=\left[\begin{array}{llll}0.589 & 0.018 & 1.045 & 0.005\end{array}\right]^{\mathrm{T}}$ ). The design and parameter estimation activities are implemented on the normalised parametric set $\boldsymbol{\Theta}$, defined as the ratio between the current parametric estimates and the true parameter values $\boldsymbol{\theta}^{0}$. Note that this 
normalisation procedure is an ideal one since in practice the true value of the parameters is unknown. However, is a simulated case study the approach is useful to have an immediate grasp of the accuracy of the estimates (in fact, it is also useful for numerical reasons; in real applications the initial guess of the parameter values can be used for normalisation).

The following design configurations have been considered and compared:

- Instance I: Conventional MBDoE;

- Instance II: OMBRE: it is assumed that $n^{u p}=1$ update may be performed and that for each manipulated input three switching levels are allowed in each updating interval; the length of each redesign time window has been fixed equal to $24 \mathrm{~h}$.

- Instance III: IDRO: in this case the iterative procedure, described in detail in section 2.3, requires to set some preliminary constants. In particular, the threshold constants $\beta, \gamma$ and $\delta$ are set to $2 / 3$; the reduction coefficient $k_{\varepsilon}$ is set to 0.9 , while the incremental coefficient $k_{w}$ is set equal to 8 . The number of scenarios $N_{s c}$ implemented in the stochastic simulation is set to 25 : this value is a good compromise between the high computational cost required by the optimization task and the necessity to simulate a reliable representation of model parameters distribution (using a computer with CPU@ 2.40GHz and RAM 8GB, IDRO requires about 5 hours of calculation whereas OMBRE needs 45 minutes; this time is required for the first design and, in fact, the calculation could be carried out before initiating the experiment; the redesign calculation time is about $1 \mathrm{~h}$ ).

It is assumed that measurements $\left(N_{s p}=8\right)$ are equidistantly distributed along the maximum experiment duration $\tau$.

Results are discussed in the following sections for the three design configurations in terms of manipulated inputs, simulated profiles and a-posteriori statistics on the final parameter estimation. 


\subsection{Instance I: conventional MBDoE}

Results in terms of predicted profiles and manipulated inputs are given in Figures $4 \mathrm{a}$ and $4 \mathrm{~d}$.Table 1 shows that the results obtained in terms of parameter precision are unsatisfactory; in fact, it is not possible to achieve a statistically sound estimation of parameter $\theta_{2}$ (Table 1 ).

\subsection{Instance II: OMBRE}

Results in terms of predicted profiles and manipulated inputs are given in Figures $4 \mathrm{~b}$ and $4 \mathrm{e}$. Also in this case, the estimation of the parameters values is quite unsatisfactory: as summarized in Table 1 parameters $\theta_{2}$ and $\theta_{3}$ cannot be estimated in a statistically satisfactory way by the end of the experimental run. Note that OMBRE is outperformed by the conventional MBDoE configuration. The initial uncertainty on model parameters is such that during the first sub-experiment it is not possible to gather sufficient information to produce an effective redesign. In fact, this issue was highlighted in the original article by the authors themselves (Galvanin et al., 2009) when they observed that in the case of poor initial information the quality of the experiments is highly affected by the updating policy and that scarcely informative redesigns could be obtained in the initial phases.

\subsection{Instance III: IDRO}

Results in terms of predicted profiles and manipulated inputs are given in Figures 4c and 4f, while the parameter estimates are given in Table 1. It is clear that, compared to MBDoE and OMBRE configuration, IDRO gives final the best estimates in terms of accuracy thanks both to the robust approach and the innovative info-based updating time optimisation which guarantees the attainment of a minimum level of information in the initial experimental phases. In fact, this is the only case where a satisfactory estimation of all parameters can be achieved within the duration on one experiment. Also note that in some case also a robust formulation for OMBRE may produce 
similarly good results, but the performance is very much dependent on the updating policy, which is dealt explicitly with only in the IDRO approach.

\section{Case study 2: A physiological model for type 1 diabetes mellitus}

This second case study considers a model of glucose homeostasis for the simulation of type 1 diabetes mellitus (Galvanin et al., 2011), based on a previous work (Lynch and Bequette, 2002). The model is described by the following set of differential and algebraic equations:

$$
\begin{aligned}
& \frac{d C_{g}}{d t}=\theta_{1} C_{g}-X\left(C_{g}+C_{g, b}\right)+\frac{D(t)}{V_{G}} \\
& \frac{d X}{d t}=\theta_{2} X+\theta_{3} I, \quad \frac{d I}{d t}=-\theta_{4}\left(I+I_{b}\right)+\frac{u(t)}{V_{I}} \\
& \frac{d C_{g s c}}{d t}=\frac{C_{g}-C_{g s c}}{5}-R_{u t} \\
& D(t)=\frac{5 A \cdot t \cdot e^{-0.05 t}}{2}, \hat{y}=C_{g s c, t o t}=C_{g s c}+C_{g s c, b}
\end{aligned}
$$

where $C_{g}$ is the blood glucose concentration $(\mathrm{mg} / \mathrm{dL}), X$ the variation of insulin concentration (mU/L) in the inaccessible compartment with respect to basal value, $I$ the variation of insulin concentration $(\mathrm{mU} / \mathrm{L})$ with respect to basal value, $u(t)$ the rate of infusion of exogenous insulin (mU/min). The measured response is the total subcutaneous glucose concentration $C_{g s c, t o t}$, modelled by Eq.(23) which represents a first-order $5 \mathrm{~min}$ lag between $C_{g}$ and $C_{g s c}$. The meal disturbances equation (24) is based on Hovorka and co-worker model (2004), with $A$ being the amount of carbohydrates of the meal, here set to be $30 \mathrm{~g}_{\mathrm{CHO}}$ (fixed). The constant basal parameter are set to the following values: the basal glucose concentrations in the blood $C_{g, b}$ and $C_{g s c, b}$ are assumed to be equal to $81 \mathrm{mg} / \mathrm{dL}$, the basal insulin concentration $I_{b}$ is $15 \mathrm{mU} / \mathrm{L}$, the glucose distribution volume $V_{G}$ is $120 \mathrm{dL}$, the insulin distribution volume $V_{I}$ is $12 \mathrm{~L}$ and the tissue rate utilization $R_{u t}$ is 0.75 $\mathrm{mg} / \mathrm{dL} / \mathrm{min}$. 
With respect to the original formulation only the inequality constraint:

$$
-y+G \leq 0
$$

is considered, where $G=70 \mathrm{mg} / \mathrm{dL}$ is the lower conservative threshold on the subcutaneous glucose concentration (y). This bound is a hard constraint not to be violated because it prevents hypoglycaemic conditions $\left(C_{g s c, t o t}<76 \mathrm{mg} / \mathrm{dL}\right)$ during real tests.

The achievable condition that characterise the experimental tests is the dilution factor $u$ (range $0 \div 115 \mathrm{mU} / \mathrm{min}$ ); this manipulated input is approximated as a piecewise constant function with $N_{s w}$ $=8$ switching intervals. It is assumed that $N_{s p}=14$ samples (equally distributed) can be taken during a $\tau=480$ min long experiment. Measurements are affected by Gaussian noise with zero mean and a standard deviation of $\sigma=5 \mathrm{mg} / \mathrm{dL}$ on $y$.

The real system is represented by the parameter set $\boldsymbol{\theta}=\left[\begin{array}{ll}0.017 & 0.0321 .540 \mathrm{E}-50.096\end{array}\right]^{\mathrm{T}}$; it is then assumed that the initial parameter estimates are affected by a $\pm 70 \%$ relative error with respect to the

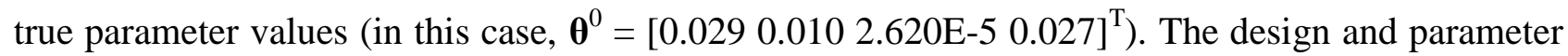
estimation activities are based on the normalised parametric set $\boldsymbol{\Theta}$, defined through the ratio between the current parametric estimates and the true parameter values.

Also in this Case study, conventional MBDoE, OMBRE and IDRO approaches have been compared:

- Instance I: Conventional MBDoE;

- Instance II: OMBRE: it is assumed that $n^{u p}=1$ update may be performed and that for each manipulated input three switching levels are allowed in each updating interval; the length of each redesign time window has been fixed equal to $240 \mathrm{~min}$.

- Instance III: IDRO: in the same way described for the first case study, the threshold constants $\beta, \gamma$ and $\delta$ are set to $2 / 3$; the reduction coefficient $k_{\varepsilon}$ is set to 0.9 , while the incremental 
coefficient $k_{w}$ is set equal to 8 . The number of scenarios $N_{s c}$ implemented in the stochastic simulation is set to 25 . The calculation time is similar to the one required for Case study 1 . Results are discussed in the following.

\subsection{Instance I: $M B D o E$}

Results in terms of predicted profiles and manipulated inputs are given in Figures 5a and 5d. The parameter estimation task proves to be totally unsatisfactory; in fact, it is not possible to achieve an accurate estimation of parameters $\theta_{1}, \theta_{2}$ and $\theta_{3}$ (Table 2).

\subsection{Instance II: OMBRE}

Results in terms of predicted profiles and manipulated inputs are given in Figures $5 \mathrm{~b}$ and $5 \mathrm{e}$. The OMBRE approach allows for a slightly better performance (Table 2), but still both parameters $\theta_{2}$ and $\theta_{3}$ cannot be estimated in a statistical satisfactory way. Once again, the behaviour reflects the initial sub-optimal design due to the initial parametric mismatch and a non-robust approach for the optimal design/redesign of the experiment.

\subsection{Instance III: IDRO}

Results in terms of predicted profiles and manipulated inputs are given in Figures $5 \mathrm{c}$ and $5 \mathrm{f}$. Table 2 shows that in this case the final results obtained in terms of parameter accuracy are greatly improved and all parameters pass the $t$-test. Note that IDRO sets the updating time exactly after 240 min like OMBRE (compare Figure 5b and Figure 5c); however, the first sub-experiment is more informative as it is explicitly designed to guarantee some level of information in a robust manner. That cannot be ensured in the OMBRE case, which is affected by the initial parametric mismatch. 


\section{Conclusions}

An innovative approach for a structured online model based redesign of the experiment (IDRO) has been presented in this paper. This technique allows for the determination of the optimal updating points at which exploiting the experimental information for the online redesigning procedure, thanks to both a robust approach and an info driven objective function to be optimised in the design phase.

This novel redesign criterion can be adopted to increase the reliability of the design task; a specific advantage over the conventional design techniques for improving parameter estimation is given by the fact that information is exploited during the experimental run; moreover, with respect to online redesign techniques (such as OMBRE), the great improvement consists in treating the design in a robust way by optimising both the redesign structure and the available information in a synergistic way.

Two distinct simulated case studies have been used to assess the effectiveness of the new technique and compare it with conventional redesign approaches: one relates to a bioreactor system, while the other one to a physiological system describing the effect of insulin on patients suffering from diabetes; results of both case studies show the higher efficiency of the proposed technique to exploit the information coming from the running experiment in an info-driven way.

Although the computational burden is still very demanding, much of the effort is required during the first calculation only, i.e. before starting the experiment. Furthermore, more tailored optimisation methods and the usage of parallel computing (especially for stochastic simulations) could improve the algorithm efficiency drastically. 


\section{Nomenclature}

General symbols

A Glucose content in the meal

$C_{g} \quad$ Blood glucose concentration

$C_{g, b} \quad$ Basal blood glucose concentration

$C_{g s c} \quad$ Subcutaneous glucose concentration

$C_{g s c, b} \quad$ Basal subcutaneous glucose concentration

$C_{\text {gsc,tot }}$ Total subcutaneous glucose concentration

$D(t) \quad$ Dynamic meal disturbance

$E(\cdot) \quad$ Expected value

$f \quad$ Differential and algebraic system implicit function

G Constraint for hypoglycaemic condition

$h \quad$ Measurements selection function

$k_{\varepsilon} \quad$ Reduction coefficient for the weight term $w_{j}$

$k_{w} \quad$ Incremental coefficient for the weight term $w_{j}$

I Variation of plasmatic insulin concentration with respect to the basal value

$I_{b} \quad$ Basal plasmatic insulin concentration

$N_{s c} \quad$ Number of scenarios

$N_{s p} \quad$ Number of samples

$N_{s w} \quad$ Number of switching for piecewise constant manipulated variables

$N_{s w, j} \quad N_{s w}$ used during the $j$-th sub-experiment

$N_{u} \quad$ Number of manipulated inputs

$N_{x} \quad$ Number of state variables

$N_{w} \quad$ Number of time invariant controls 
$N_{y} \quad$ Number of measured variables

$N_{\theta} \quad$ Number of model parameters

$N_{C} \quad$ Number of constraints

$N_{D} \quad$ Number of constraints on estimated outputs

$N_{E} \quad$ Number of constraints on unmeasured state variables

$n_{s w} \quad$ Number of switching levels

$n^{u p} \quad$ Number of redesigns

$n_{\varphi} \quad$ Number of design variables

$n_{\varphi, i} \quad$ Number of design variables for the $i$-th redesign

$r \quad$ Growth rate function for bioreactor model

$R_{u t} \quad$ Tissue rate utilization

$s_{i j} \quad i j$-th element of the inverse matrix of measurements errors

$t \quad$ Time

$t(\cdot) \quad t$-value distribution

$t_{i} \quad i$-th $t$-value

$t^{\text {up } \quad \text { Updating time point }}$

$t^{\mathrm{up}, i} \quad$ Updating time point before the $i$-th redesign

$t_{1-\alpha}^{r e f} \quad$ Reference $t$-value at (1- $\left.\alpha\right) \%$ of confidence

$t_{s p, i} \quad i$-th sampling time

$u \quad$ Generic manipulated input

$v_{i j} \quad i j$-th element of the parametric variance-covariance matrix

$V_{G} \quad$ Glucose distribution volume

$V_{I} \quad$ Insulin distribution volume

$w_{j} \quad$ Weight term for IDRO objective function calculation in $(13,14)$ 
$x \quad$ Generic state variable

$X \quad$ Variation of insulin concentration in the sub-compartment with respect to the basal value

y $\quad$ Generic measured output

$\alpha \quad$ Statistical degree of significance

$\beta \quad$ Costant term for $t^{u p}$ threshold definition

$\gamma \quad$ Costant term for $N_{s w}$ threshold definition

$\delta \quad$ Costant term for $N_{s p}$ threshold definition

$\varphi_{i} \quad i$-th element of the design vector

$\theta_{i} \quad i$-th model parameter

$\hat{\theta}_{i} \quad i$-th estimated model parameter

$\sigma \quad$ Standard deviation on measured variables

$\hat{\sigma}_{\theta i} \quad$ Standard deviation on the $i$-th estimated model parameter

$\tau \quad$ Experiment/Test duration

$\psi \quad \mathbf{V}_{\theta} / \mathbf{H}_{\theta}$ measurement function for design optimization

Vectors and Matrices [dimension]

C Set of constraint functions $\left[N_{C}\right]$

G Set of active constraints $\left[N_{C}\right]$

$\mathbf{H}_{\theta} \quad$ Dynamic information matrix $\left[N_{\theta} \times N_{\theta}\right]$

$\mathbf{H}_{\theta}^{0} \quad$ Preliminary information matrix $\left[N_{\theta} \times N_{\theta}\right]$

$\mathbf{H}_{\theta \mid j} \quad$ Information matrix for the $j$-the redesign $\left[N_{\theta} \times N_{\theta}\right]$

L Constant information matrix of (11) $\left[N_{\theta} \times N_{\theta}\right]$

$\mathbf{M}_{k} \quad$ Information matrix for the $k$-th sample $\left[N_{\theta} \times N_{\theta}\right]$

$\mathbf{y}_{0} \quad$ Vector of initial conditions $\left[N_{y}\right]$ 


\begin{tabular}{|c|c|}
\hline $\mathbf{y}$ & Measurements vector $\left[N_{y}\right]$ \\
\hline$\hat{\mathbf{y}}$ & Vector of estimated responses $\left[N_{y}\right]$ \\
\hline $\mathbf{t}^{s p}$ & Vector of sampling points $\left[n_{s p}\right]$ \\
\hline $\mathbf{u}$ & Vector of manipulated inputs $\left[N_{u}\right]$ \\
\hline $\mathbf{V}_{\theta}$ & Variance-covariance matrix of model parameters $\left[N_{\theta} \times N_{\theta}\right]$ \\
\hline $\mathbf{w}$ & Vector of time-invariant control $\left[N_{w}\right]$ \\
\hline $\mathbf{x}$ & Vector of state variables $\left[N_{x}\right]$ \\
\hline$\hat{\mathbf{x}}_{k}$ & Vector of states estimation at the $k$-th instant $\left[N_{x}\right]$ \\
\hline $\mathbf{x}^{0}$ & Vector of initial states $\left[N_{x}\right]$ \\
\hline$\dot{\mathbf{x}}$ & Vector of derivatives on state variables $\left[N_{x}\right]$ \\
\hline$\varphi$ & Design vector $\left[n_{\varphi}\right]$ \\
\hline$\varphi_{j}$ & Design vector before the $j$-th update $\left[n_{\varphi, j}\right]$ \\
\hline $\boldsymbol{\theta}$ & Vector of true model parameters for the system $\left[N_{\theta}\right]$ \\
\hline$\hat{\boldsymbol{\theta}}$ & Vector of estimated values of model parameters $\left[N_{\theta}\right]$ \\
\hline $\boldsymbol{\theta}^{0}$ & Vector of initial guesses of model parameters $\left[N_{\theta}\right]$ \\
\hline $\boldsymbol{\Theta}$ & Vector of normalized model parameters for the subject $\left[N_{\theta}\right]$ \\
\hline$\Sigma_{y}$ & Variance-covariance matrix of measurement errors $\left[N_{y} \times N_{y}\right]$ \\
\hline $\boldsymbol{\Sigma}_{\theta}$ & Prior information matrix $\left[N_{\theta} \times N_{\theta}\right]$ \\
\hline
\end{tabular}

Acronyms

AID

DAEs Differential and algebraic equations system

DE-OMBRE OMBRE including disturbance estimation

IDRO Information driven redesign optimisation 
NLPSQP Nonlinear programming sequential quadratic approach

MBDoE Model-based design of experiments

OMBRE Online model-based redesign of experiments

\section{References}

Asprey SP, Macchietto S. Statistical Tools for Optimal Dynamic Model Building. Comput Chem Eng 2000; 24:1261-1267.

Asprey SP, Macchietto S. Designing robust optimal dynamic experiments. J Process Contr 2002; 12:545-556.

Bard, Y. Nonlinear Parameter Estimation. New York: Academic Press Inc; 1974.

Barz T, López Cárdenas DC, Arellano-Garcia H, Wozny G. Experimental evaluation of an approach to online redesign of experiments for parameter determination. AIChE J 2013; 59: 1981-1995.

Bernaerts K, Van Impe JF. Optimal dynamic experiment design for estimation of microbial growth kinetics at sub-optimal temperatures: Modes of implementation. Sim Model Pract Th 2005; 13:129-138.

Chakrabarty A, Buzzard GT, Rundell, AI. Model-based design of experiments for cellular processes. Wiley Interdiscip Rev Syst Biol Med 2013; 5(2):181-203.

Espie D, Macchietto S. The Optimal Design of Dynamic Experiments AIChE J 1989; 35:223-229.

Franceschini G, Macchietto S. Model-based design of experiments for parameter precision: State of the art. Chem Eng Sci 2008; 63:4846-4872.

Galvanin F, Barolo M, Bezzo F. Towards on-line model-based design of experiments. In: Computer-Aided Chemical Engineering 24, 18th European Symp. on Computer Aided Process Engineering (B. Braunschweig and X. Joulia, Eds.), Elsevier, Amsterdam (The Netherlands); 2008. p. $349-354$ 
Galvanin F, Barolo M, Bezzo F. Online model-based redesign of experiments for parameter estimation in dynamic systems. Ind Eng Chem Res 2009; 48:4415-4427.

Galvanin F, Barolo M, Pannocchia G, Bezzo F. Online model-based redesign of experiments with erratic models: a disturbance estimation approach. Comput Chem Eng 2012; 42:138-151.

Galvanin F, Boschiero A, Barolo M, Bezzo F. Model-based design of experiments in the presence of continuous measurements systems. Ind Eng Chem Res 2011; 50:2167-2175.

Galvanin F, Macchietto S, Barolo M, Bezzo F. Optimal design of clinical tests for the identification of physiological models of type 1 diabetes mellitus. Ind Eng Chem Res 2009a; 48:1989-2002.

Gerencser L, Hjalmarsson H, Martensson J. Identification of ARX systems with non-stationary inputs - asymptotic analysis with application to adaptive input design. Automatica 2009; 45: 623-633.

Houska B, Telen D, Logist F, Diehl M, Van Impe J. F. M. An economic objective for the optimal design of nonlinear dynamic processes. Automatica 2015; 51: 98-103.

Körkel S, Kostina E, Bock HG, Schlöder JP. Numerical methods for optimal control problems in design of robust optimal experiments for nonlinear dynamic processes. Optim Method Softw 2004; 19:327-338.

Lindqvist $\mathrm{K}$, Hjalmarsson $\mathrm{H}$. Identification for control: adaptive input design using convex optimisation. In: Proc. 40th IEEE Conference on Decision and Control; Orlando, Florida, USA; 2001.

Lynch SM, Bequette BW. Model predictive control of blood glucose in type I diabetics using subcutaneous glucose measurements. In: Proc. American Control Conference; Anchorage, AK, USA; 2002.

Mehra RK. Optimal input signals for parameter estimation in dynamic systems: survey and new results. IEEE T Automat Contr 1974; 19:753-768. 
Munack A, Posten C. Design of optimal dynamical experiments for parameter estimation. In: Proceedings of the American Control Conference, Pittsburgh, USA; 1989. p. 2011-2016.

Prasad V, Vlachos DG. Multiscale model and informatics-based optimal design of experiments: application to the catalytic decomposition of ammonia on ruthenium. Ind Eng Chem Res 2008; 47:6555-6567.

Pukelsheim F. Optimal Design of Experiments. J. Wiley \& Sons, New York, USA; 1993.

Schenkendorf R, Mangold M. Online model selection approach based on Unscented Kalman Filtering. J Process Contr 2013; 23:44-57.

Stigter JD, Vries D, Keesman KJ. On adaptive optimal input design: a bioreactor case study. AIChE J 2006; 52:3290-3296.

Zullo L. Computer aided design of experiments. An engineering approach. Ph.D. Thesis, The University of London, UK; 1991. 


\section{Table Captions}

Table 1 Case study 1-Fermentation model: A-optimal MBDoE, OMBRE and IDRO. Results from final parameter estimation including standard deviation and t-value $95 \%$ of the normalized parametric set. Double asterisks denote $t$-values failing the t-test.

Table 2 Case study 2- Diabetes model: A-optimal MBDoE, OMBRE and IDRO. Results from final parameter estimation including standard deviation and t-value $95 \%$ of the normalized parametric set. Double asterisks denote $t$-values failing the $t$-test. 


\section{Figure Captions}

Figure 1 Flowchart of OMBRE procedure

Figure 2 The logic of IDRO approach applied to a generic two-parameters model: the red solid stepwise profile represents the dynamic weighted $t_{1-\alpha}^{\text {ref }}$ profile (at each $t^{u p, j}$ the weight $w_{j-1}$ is modified, as described in Figure 3 ). The black solid and dashed stepwise profiles represent the parametric t-values of the two model parameters, whose values increase with the number of collected samples. The dash-dot vertical lines represent the updating times $t^{u p, j}$ for the experimental run.

Figure 3 Flowchart of IDRO procedure

Figure 4 Case study 1- Fermentation model: results in terms of predicted profiles for biomass $\left(y_{1}\right)$ and substrate ( $\left.y_{2}\right)$ concentration for a) A-optimal MBDoE, b) OMBRE, c) IDRO: the experimental samples are indicated by circles $\left(y_{1}\right)$ and triangles $\left(y_{2}\right)$ with error bars. Optimal profiles for the dilution factor $\left(u_{1}\right)$ and the substrate concentration in the feed $\left(u_{2}\right)$ are reported for d) A-optimal MBDoE, e) OMBRE, f) IDRO.

Figure 5 Case study 2-Diabetes model: results in terms of predicted profiles for biomass $\left(y_{1}\right)$ and substrate $\left(y_{2}\right)$ concentration for a) A-optimal MBDoE, b) OMBRE, c) IDRO: the experimental samples are indicated by circles ( $\left.y_{1}\right)$ and triangles $\left(y_{2}\right)$ with error bars. Optimal profiles for the dilution factor $\left(u_{1}\right)$ and the substrate concentration in the feed $\left(u_{2}\right)$ are reported for d) A-optimal MBDoE, e) OMBRE, f) IDRO. 


\section{Tables}

Table 1

\begin{tabular}{|c|c|c|c|c|c|c|}
\hline & & $\begin{array}{c}\text { Normalized } \\
\text { Model Parameter }\end{array}$ & $\begin{array}{c}\text { Real } \\
\text { value }\end{array}$ & $\begin{array}{l}\text { Final } \\
\text { value }\end{array}$ & $\begin{array}{l}\text { Standard } \\
\text { deviation }\end{array}$ & $t$-value $95 \%$ \\
\hline & & $\Theta_{1}$ & 1 & 1.016 & 0.017 & 28.17 \\
\hline Conve & tional MBDoE & $\Theta_{2}$ & 1 & 0.544 & 0.325 & $0.769 * *$ \\
\hline Conve & IIIOHaA MIDDOE & $\Theta_{3}$ & 1 & 1.046 & 0.025 & 18.94 \\
\hline & & & & & & \\
\hline & & & & & Reference $t$-value $95 \%$ & 1.783 \\
\hline & & $\Theta_{1}$ & 1 & 2.304 & 17.61 & $0.047 * *$ \\
\hline & & $\Theta_{2}$ & 1 & 3.136 & 32.70 & $0.035 * *$ \\
\hline & $1^{\text {st }}$ sub-experiment & $\Theta_{3}$ & 1 & 1.013 & 0.037 & 9.925 \\
\hline & & $\Theta_{4}$ & 1 & 1.028 & 0.095 & 3.905 \\
\hline OMRRF & & & & & Reference $t$-value $95 \%$ & 2.132 \\
\hline OMBКЕ & & $\Theta_{1}$ & 1 & 2.528 & 20.42 & $0.057 * *$ \\
\hline & & $\Theta_{2}$ & 1 & 2.742 & 27.77 & $0.045 * *$ \\
\hline & $2^{\text {nd }}$ sub-experiment & $\Theta_{3}$ & 1 & 0.988 & 0.034 & 13.19 \\
\hline & & $\Theta_{4}$ & 1 & 0.948 & 0.088 & 4.92 \\
\hline & & & & & Reference $t$-value $95 \%$ & 1.783 \\
\hline & & $\Theta_{1}$ & 1 & 1.042 & 0.043 & 9.959 \\
\hline & & $\Theta_{2}$ & 1 & 0.779 & 0.435 & $0.731 * *$ \\
\hline & $1^{\text {st }}$ sub-experiment & $\Theta_{3}$ & 1 & 1.053 & 0.060 & 7.196 \\
\hline & & $\Theta_{4}$ & 1 & 1.242 & 0.213 & 2.382 \\
\hline IDRO & & & & & Reference $t$-value $95 \%$ & 1.943 \\
\hline IDKU & & $\Theta_{1}$ & 1 & 0.999 & 0.013 & 34.72 \\
\hline & & $\Theta_{2}$ & 1 & 0.912 & 0.145 & 2.88 \\
\hline & $2^{\text {nd }}$ sub-experiment & $\Theta_{3}$ & 1 & 1.003 & 0.023 & 20.29 \\
\hline & & $\Theta_{4}$ & 1 & 1.020 & 0.079 & 5.917 \\
\hline & & & & & Reference $t$-value $95 \%$ & 1.783 \\
\hline
\end{tabular}


Table 2

\begin{tabular}{|c|c|c|c|c|c|c|}
\hline \multirow{6}{*}{\multicolumn{2}{|c|}{ Conventional MBDoE }} & $\begin{array}{c}\text { Normalized } \\
\text { Model Parameter }\end{array}$ & $\begin{array}{l}\text { Real } \\
\text { value }\end{array}$ & $\begin{array}{l}\text { Final } \\
\text { value }\end{array}$ & $\begin{array}{l}\text { Standard } \\
\text { deviation }\end{array}$ & $t$-value $95 \%$ \\
\hline & & $\Theta_{1}$ & 1 & 1.127 & 0.057 & $1.660 * *$ \\
\hline & & $\Theta_{2}$ & 1 & 1.358 & 1.190 & $0.741 * *$ \\
\hline & & $\Theta_{3}$ & 1 & 1.648 & 0.037 & $0.474 * *$ \\
\hline & & $\Theta_{4}$ & 1 & 1.019 & 0.082 & 2.955 \\
\hline & & & & & Reference $t$-value $95 \%$ & 1.812 \\
\hline \multirow{10}{*}{ OMBRE } & \multirow{5}{*}{$1^{\text {st }}$ sub-experiment } & $\Theta_{1}$ & 1 & 0.654 & 0.260 & $0.793 * *$ \\
\hline & & $\Theta_{2}$ & 1 & 0.586 & 0.678 & $0.272 * *$ \\
\hline & & $\Theta_{3}$ & 1 & 0.388 & 0.565 & $0.216 * *$ \\
\hline & & $\Theta_{4}$ & 1 & 0.312 & 0.920 & $0.107 * *$ \\
\hline & & & & & Reference $t$-value $95 \%$ & 2.354 \\
\hline & \multirow{5}{*}{$2^{\text {nd }}$ sub-experiment } & $\Theta_{1}$ & 1 & 1.019 & 0.185 & 2.478 \\
\hline & & $\Theta_{2}$ & 1 & 1.657 & 0.560 & $1.327 * *$ \\
\hline & & $\Theta_{3}$ & 1 & 1.586 & 0.848 & $0.840 * *$ \\
\hline & & $\Theta_{4}$ & 1 & 1.067 & 0.143 & 3.352 \\
\hline & & & & & Reference $t$-value $95 \%$ & 1.812 \\
\hline \multirow{10}{*}{ IDRO } & \multirow{5}{*}{$1^{\text {st }}$ sub-experiment } & $\Theta_{1}$ & 1 & 1.059 & 0.116 & 2.873 \\
\hline & & $\Theta_{2}$ & 1 & 1.339 & 0.582 & $\mathbf{0 . 7 2 3} * *$ \\
\hline & & $\Theta_{3}$ & 1 & 1.436 & 0.522 & $0.865 * *$ \\
\hline & & $\Theta_{4}$ & 1 & 1.044 & 0.256 & $1.282 * *$ \\
\hline & & & & & Reference $t$-value $95 \%$ & 2.354 \\
\hline & \multirow{5}{*}{$2^{\text {nd }}$ sub-experiment } & $\Theta_{1}$ & 1 & 1.106 & 0.055 & 9.035 \\
\hline & & $\Theta_{2}$ & 1 & 1.034 & 0.170 & 2.736 \\
\hline & & $\Theta_{3}$ & 1 & 1.247 & 0.169 & 3.322 \\
\hline & & $\Theta_{4}$ & 1 & 1.082 & 0.073 & 6.625 \\
\hline & & & & & Reference $t$-value $95 \%$ & 1.812 \\
\hline
\end{tabular}


Figures

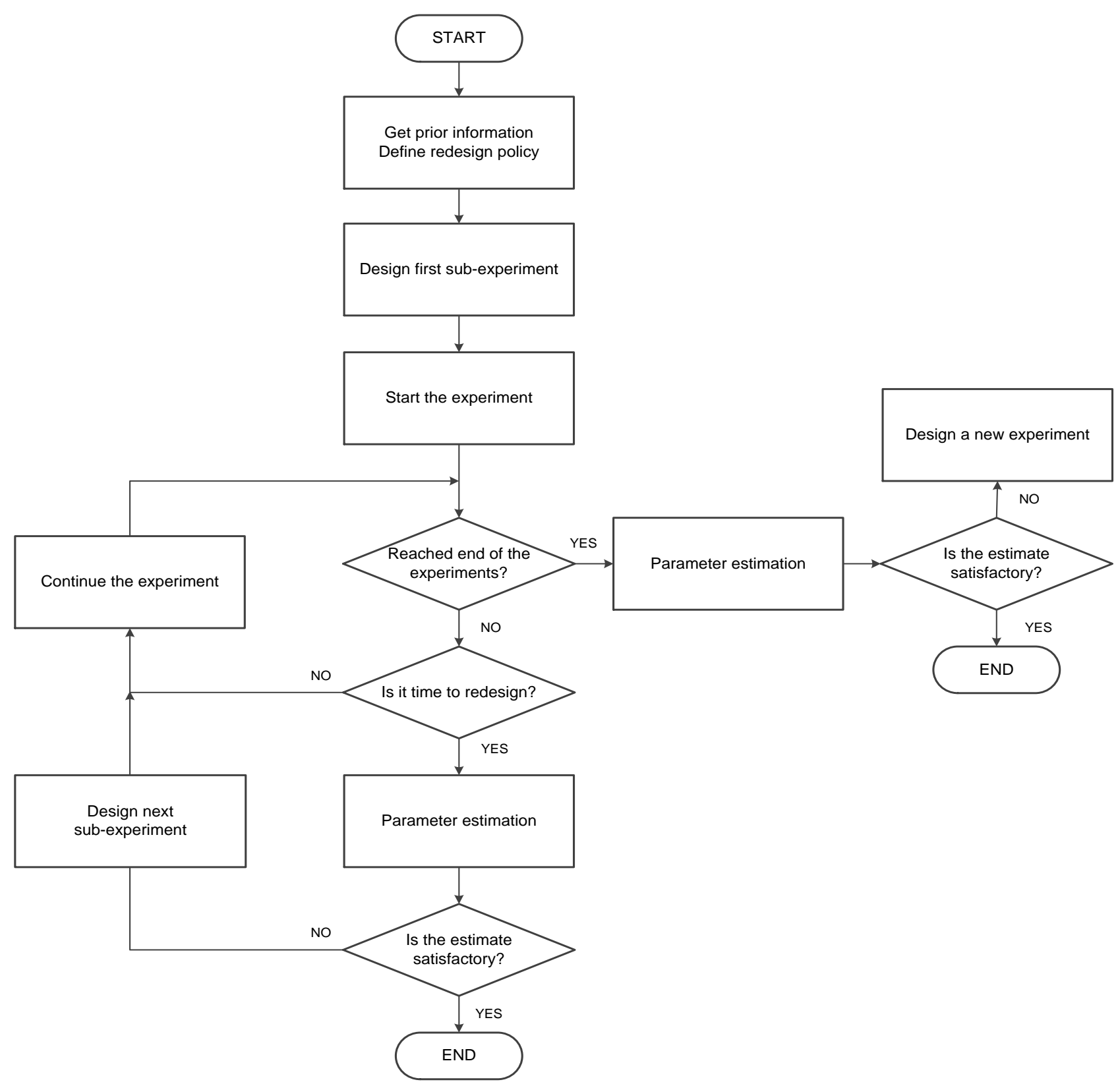

Figure 1 


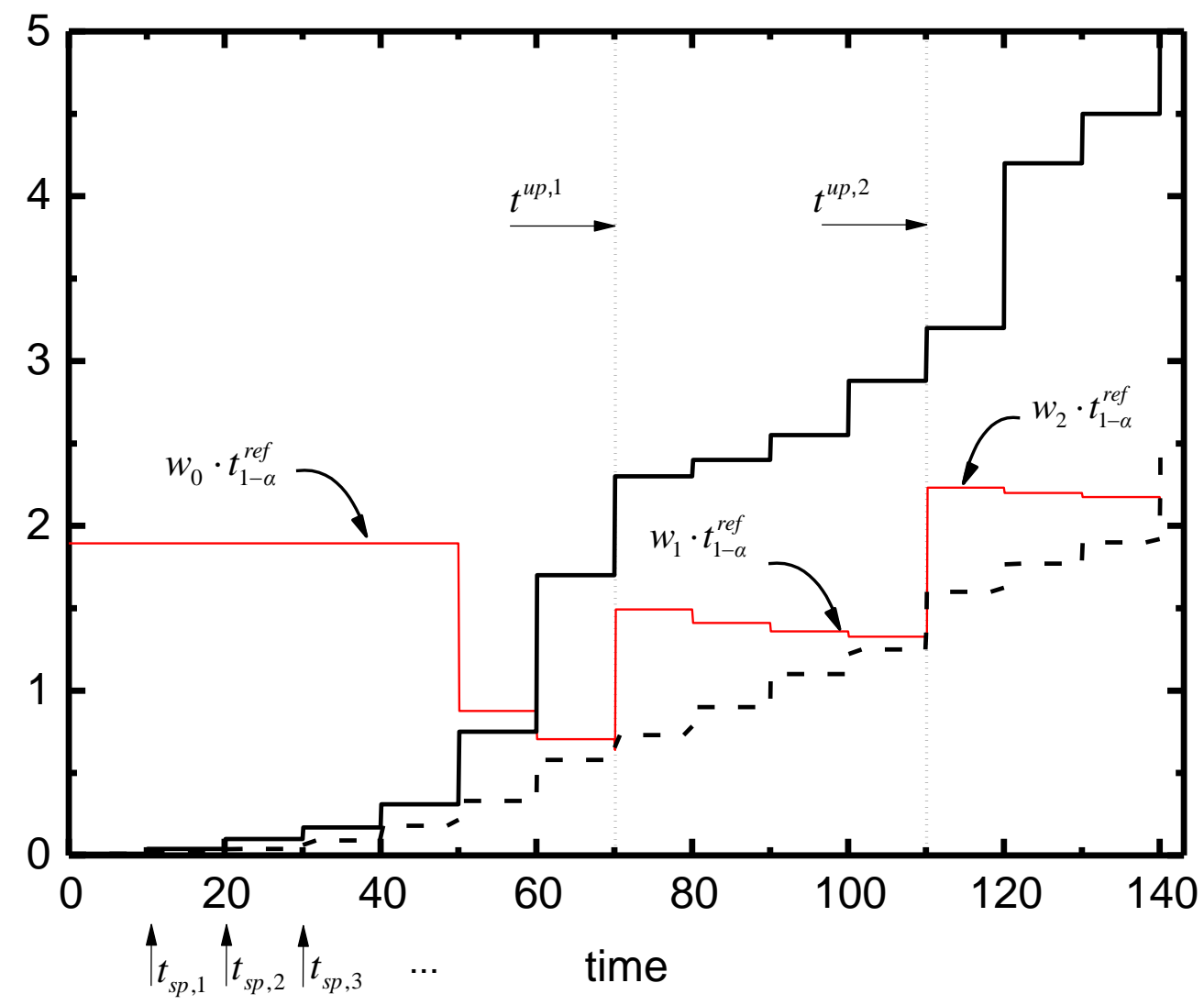

Figure 2 


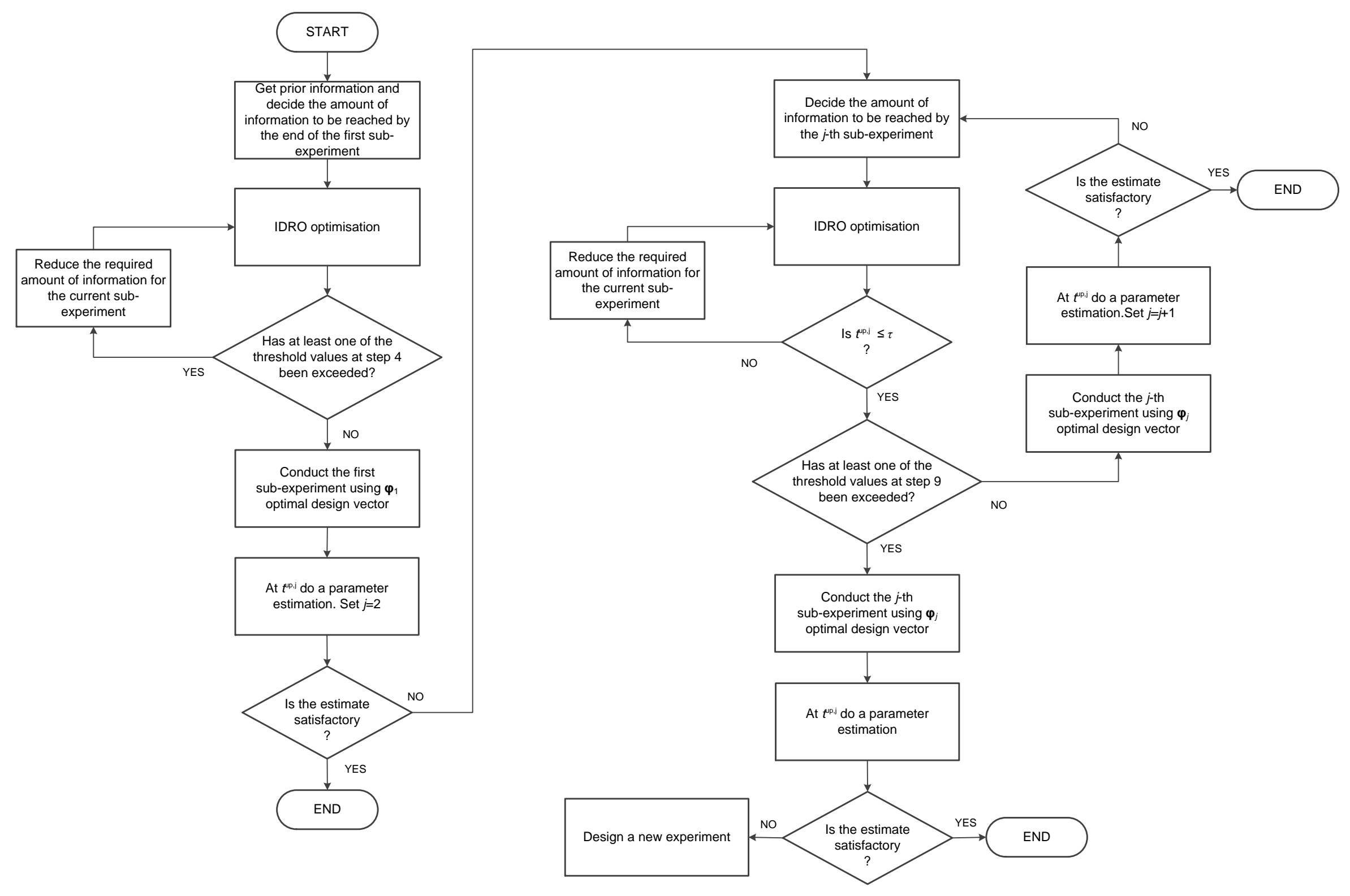

Figure 3 

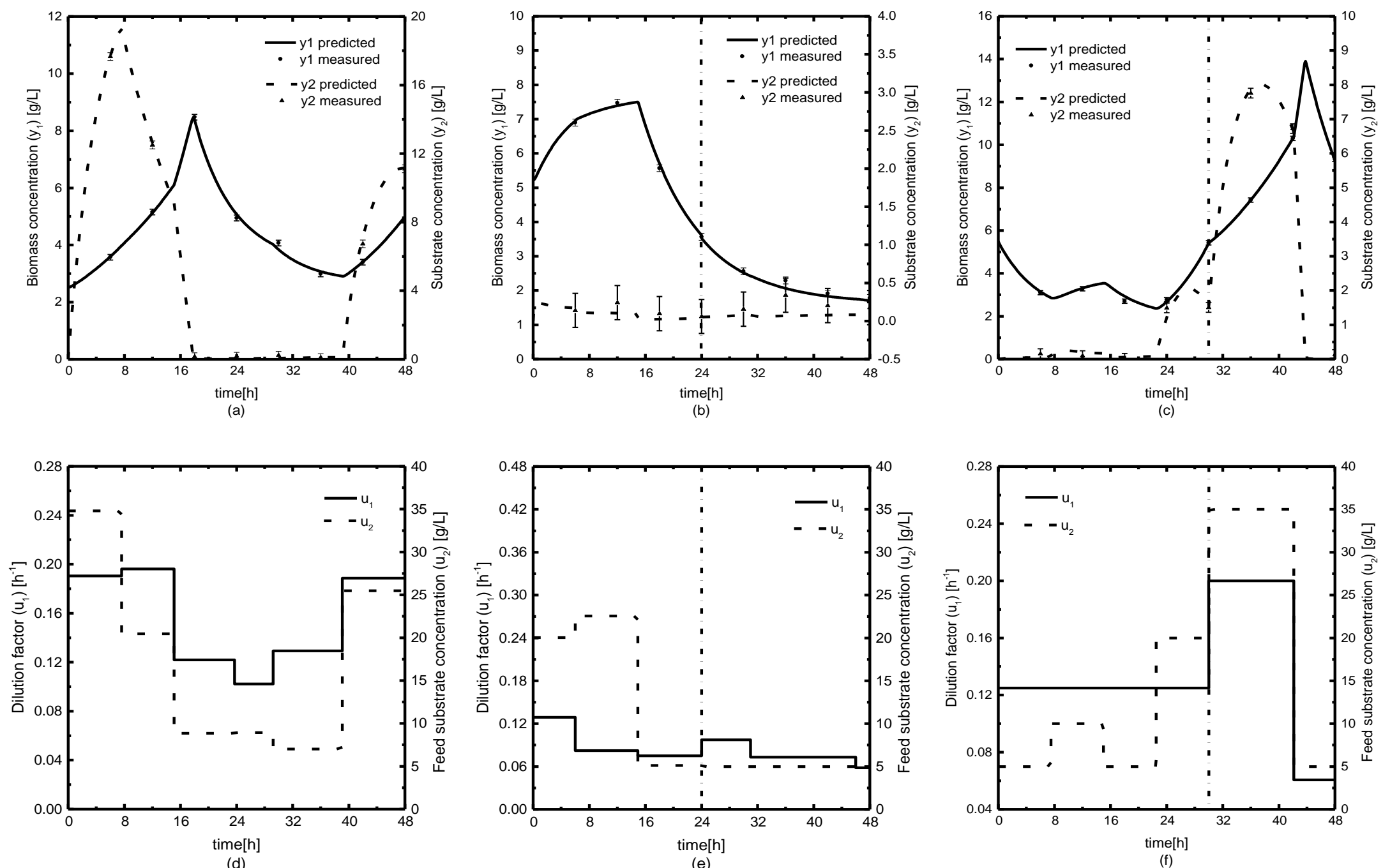

Figure 4 

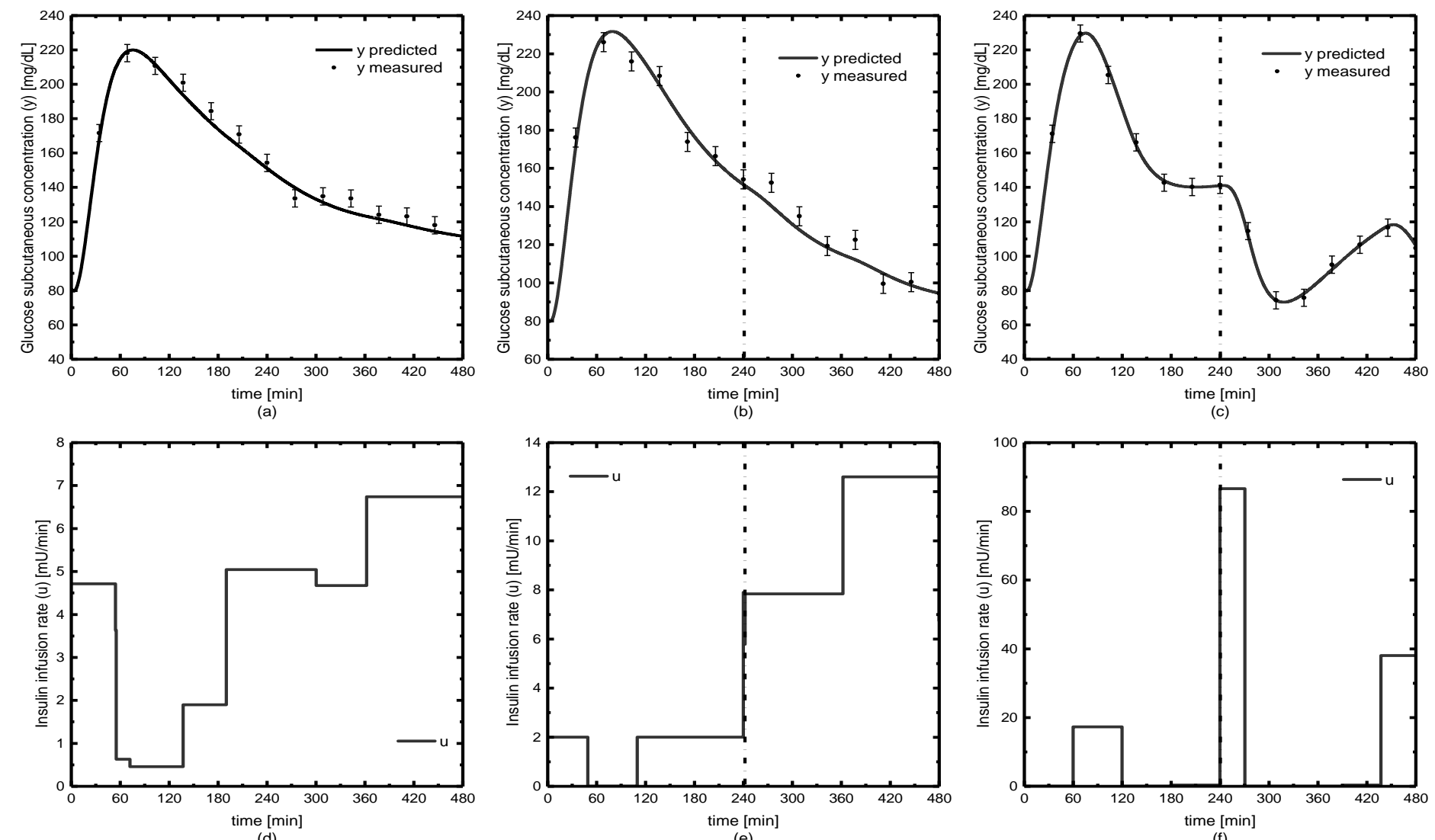

Figure 5 
